ionischen Zustand in charakteristischer Weise aus. So konnte durch Quellung in Ammoniak der Leimgehalt einer Gelatine auf 0,6 Proz. heruntergebracht werden, wobei der feste Aggregatzustand infolge der Wasserbindung als Hydrat erhalten blieb. während eine neutrale Gelatine unterhalb 1,5 Proz. unter den gleichen Verhältnissen nur in flüssiger Form zu gewinnen ist. Ebenso war es uns möglich, typische Eiweißsole durch Umwandlung in ionisches EiweiB als feste Gallerten zu erhalten. - Es bestehen also innige Beziehungen zwischen osmotischem Druck und Quellungsdruck bei den Eiweiß-Körpern. Die Kräfte, die in beiden Fällen wirksam werden, sind in ihrem Hauptanteil identisch und nur die Anordnungen, durch welche die Eiweißteilchen im Osmometer und in der Gallerte zusammengehalten sind, erscheinen verschieden. Ein Unterschied zwischen Quellung und Hydratation in Lösung läßt sich nicht angeben, da alle Beobachtungen hier und dort für eine Bindung des Wassers nicht nach stöchiometrischen Verhältnissen sprechen.

\title{
Rotes Platin als Analogon des Cassius'schen Goldpurpurs.
}

\author{
Von Lothar Wohler; nach Versuchen, gemeinsam mit A. Spengel.
}

(Mitteilung aus dem Chemischen Institut der Technischen Hochschule in Karlsruhe.1)

(Fingegangen an 7. September 1910)

Bei meinen Versuchen über die Oxydation metallischen Platins, sowie über die Platinoxyde und ihre Verbindungen, wurde als zuverlässigste Reaktion auf Platin ${ }^{2}$ ) die bekannte Rotfärbung gefunden, welche salzsaures Zinnchlorür mit Platinlösungen erzeugt. Die Empfindlichkeit ist so grob, daB $10^{-7} \mathrm{~g}$ Pt im Kubikzentimeter Flüssigkeit noch eben durch Gelbfärbung erkennbar ist. Ueber die Ursache der Färbung findet sich in der analytischen Literatur nicht viel. Einige Lehrbücher ${ }^{3}$ ) führen die Rotfärbung auf Reduktion zu dem in gröBerer Konzentration dunkelroten $\mathrm{PtCl}_{4}$ " zurück. Diese Annahme ist schon deshalb unrichtig, weil auch geringe Mengen $\mathrm{PtCl}_{4}{ }^{\prime \prime}$ in farbloser Lösung mit Zinnchlorür die Rotfärbung, bei Spuren Gelbfärbung, erzeugen. Es liegt nahe, an einen undissoziierten Komplex von $\mathrm{PtCl}_{4}{ }^{\prime \prime}$ mit $\mathrm{Sn}^{\prime \prime}$ zu denken $-\mathrm{SnCl}_{4}$ erzeugt die Reaktion nicht ..., ähnlich dem roten Fe(CNS)g. In der Tat fanden wir, dak wie bei diesem auch bei jenem sich die Rotfärbung durch Aether, viel besser noch durch Aethylazetat, der Lösung entziehen läbt, ein Zeichen für geringe Dissoziation. Da aber auch Platinchlorid und Zinnchlorid in Essigester und Aether löslich sind, so konnte die Untersuchung dieser Lösungen keinen AufschluB über die Zusammensetzung der Rotfärbung geben. Beim

1) Auszugsweise mitget. auf der 79. Naturf.Vers. 1907; vg1. Koll. - Zeitschr. 2, Süppl. 1, III (1907).

2) Fbenso empfindlich, aber weniger zuverlässig ist die Rotfärbung durch Jodkaliurn (F i eld, Chen. News 43, 75).

3) R. Fresenius, Qualit. Analyse 1895: Miller und Kiliani, 3. Aufl., 261; F. P. Treadwell, Qualitative Analyse (1904), 218.
Verdunsten der Lösung im Vakuum bleibt neben den farblosen Kristallen von Zinnchlorür und -chlorid eine gelbrote gelatinöse Masse, die in wenig Wasser und Salzsäure sich wieder löst. Direkt um die Kristalle herum aber ist dic Grundmasse tief dunkelrot, und beim Erwärmen scheidet sich auf den Kristallen von Zinnchlorür metallisches Platin braun bis schwary $a b$, das sich nun nicht mehr im Wasser lösen läbt. Die dunkelrote Masse wird auch ohne Erwärmen allmählich braunrot, bleibt aber löslich in Wasser und Salzsäure, wie die umgebende gelbrote, so daß sich alle Farbenabstufungen von gelbrot bis schwarz nach den Kristallen zu zeigen.

Beim Eingießen der roten Essigesterlösung in Wasser entsteht ein schokoladebrauner Niederschlag chlorhaltigen amorphen Oxydes, ähnlich demjenigen, der sich langsam bildet beim starken Verdünnen der wässerigen roten Lösung. Aus saurer Lösung scheidet sich so fort nichts ab, wohl aber nach längerem Stehen an der Luft. $\mathrm{Da}$ dieser Niederschlag nach dem Abfiltrieren und Auswaschen sich in Salzsäure wieder mit roter Farbe löst, zur Lösung gleicher Eigenschaften wie vorher, so konnte vielleicht aus seiner Zusammensetzung auf die des angenommenen komplexen Chlorids geschlossen werden.

Nun hat bereits R. Schneider) den braunschwarzen Niederschlag untersucht, der beim Versetzen von einprozentiger Platinlösung mit Zinnchlorür unter Luftabschlub nach Neutralisation des Gemisches mit Ammoniak ent-

9) Poggendorfi's Ann. 136, 105 (1869). 
steht. Er gibt ihm die Forme! eines zinnsauren Zinnoxydul-Platinsuboxyds, $\mathrm{Pi}_{3} \mathrm{Sn}_{6} \mathrm{O}_{40}$, und findet in Uebereinstimmung damit, $\mathrm{daB}$ die Rotfärbung am tiefsten bei 3 Atomen Zirn auf 1 Grammatom Platin ist; daB dann nach der Ausfällung auch kein Platin mehr in Lösung sich befindet, wohl aber bei Zusatz von veniger Zinnchlorür, und er formuliert daher den Vorgang oxydisch:

$2 \mathrm{PtO}_{2}+6 \mathrm{SnO}=\mathrm{Pt}_{2} \mathrm{O}+3 \mathrm{Sn}_{2} \mathrm{O}_{3}=\mathrm{Pt}_{2} \mathrm{Sn}_{6} \mathrm{O}_{10}$ Schon hier sei einschaltend bemerkt, das mir der Versuch zeigte, Jaß die Furbticfe der roten Losung keineswegs stöchiometrisch definiert ist. sodab ilure Verwendung zur kolorimetrischen Bestimmung des Platins, wie sie nach $S$ chneider angenommen werden könnte, und daher für diese $Z$ wecke auch vorgeschlagen wurde ${ }^{5}$ ), nicht angängig ist. Als ich nämlich 1 Pt als Chlorür mit $3 \mathrm{Sn}$ als Chlorär in einer mit Kohlensüure gefüllten Stöpselflasche mischte und unter Luftabschlub 14 Tage stehen lieb, wurde die intensiv rot gefärbte Lösung fortdauernd dunkler bis zur Undurchsichtigkeit. Nach weiteren drei Wochen war bei einer Temperatur von $40 \mathrm{hjs}$ $50^{\circ}$ die klare Losung beinahe schwarz und getrübt durch eine braune sehr fein verteilte Suspension, die sich auch nach weiterem ruhigen Stehen während mehr als 2 Jahren kaum in Spuren abgesetzt hatte, vielmehr den Eindruck schwarzbraunen kolloiden Platins machte.

Fügt man unter LuftabschluB in Kohlendioxyd auf 1 Grammatom Platin nur 1 Grammatom Zimn als Chorür, so tritt nur eine Gelbfärbung auf, bereits bei 2 Grammolen Zinuchlorür wird die Lösung aber undurchsichtig blutrot, die auch bei weiterer Zufïgung von insgesamt 3 und 4 Grammolen nicht verändert aussieht. Bei sofortiger Zufügung dieser größeren Menge Zinnchlorür ist die Färbung allerdings heller, wird aber beim Stehen, schnell beim Erwärmen, leicht dunkelrot. Auf die bereits entstandene tief dunkelrote Färbung dagegen ist ein Ueberschub s of ort ohne EinfluB. Beim Stehen werden aber alle diese Lösungen viel dunkler, woraus hervorgeht, daB die Reaktion vollständig nur langsam verläuft. Dies wird durch folgendeVersuche bestätigt. Fügt man nach Schneider's Vorschrift zu 1 Grammol Platinchlorid weniger als 3 Grammole Zinnchlorür -- es wurden 2,5 Grammole langsam zugesetzt - erwärmt zur Beschleunigung der Reaktion auf dem Wasserbade und gibt

5) L. Wohler, Ber. d. Deutsch. chem. Ges. 36, 3483 (1903); R. Ruef, Zeitschr. f. Elektr. 14, 310 (1908). dann erst Ammoniak hinzu, so ist das Filtrat entgegen Schneider frei von Platin. Fügt man dagegen 3 Mole Zinnchlorür sofort und kalt zu 1 Grammol Platinchlorid und fält sofort mit Ammoniak, so befindet sich Platin noch unverändert im Filtrat infolge zu laigsamer Reaktion.

Dies in Verbindung damit, daß Zinnsesquioxyd oder seine Derivate bisher nicht bekannt sind ${ }^{6}$ ), nach früheren Tensionsuntersuchungen ${ }^{7}$, an Palladiumoxydul ein Palladiumsuboxyd nicht existiert, so daB auch ein Platinsuboxyd recht unwahrs heinlich ist, machte die erneute Untersuchung des Schneider'schen schwarzen Niedershlags notwendig. Hergestellt wurde die Substanz (als Nr. 1 bezeichnet) einmal aus heiBem Kaliumchloroplatinat mit fast neutralem Zinnchlorür, bis der sofort entstehende braune Niederschlag sich nicht melar vermehrte. Er wurde dann oftmals kochend heiß dekantiert zur Befreiung von Chlorid, das darin chemisch gebunden zu sein scheint, oder als Absorptionsverbindung, die gerade Metazinnsäure besonders stark mit Salzsăure bildet ${ }^{8}$ ). Die vorher grobflockige Fällung wird dabei dunkier und pulvrig, sie verliert ihre leichte Löslichkeit in verdïnnter Salzsäure, bleibt aber in konzentrielter Salzsüure ungetrocknet löslich, getrocknet nur zuweilen noch, zeigt also das charakteristische Verhalten des Alterns kolloider Metalloxyde, wie es an den Oxyden des Platins und des Palladiums besonders deutlich beobachtet ist ${ }^{9}$ ). Eine andere Substanz $(\mathrm{Nr}$. 2) wurde durch Hydrolyse infolge starken Verdünnens der sauren Lösungen gefällt, eine weitere ( $\mathrm{Nr} .3$ ) aus Platinchlorid durch Ausziehen der roten Lösung mit Pssigester und durch Eingießen desselben in viel Wasser. Die letztere Fallung war heller braun und lieb sich schneller chlorfrei erhalten beim Auskochen mit Wasser. Im Verhältnis 1:6 waren die beiden Metalle angewandt worden bei der Herstellung einer anderen Fällung (Nr. 4) aus Platinchlorid und Zinnchlorür durch einfache Hydrolyse unter Luftabschlub; in Verhältnis $1: 3$ aus Platinchlorür, und zwar unter Luftabschlub durch Ausfallung mit Ammoniak in einer weiteren Substanz (Nr. 5). Die exsikkator-

9) Die von E. A. Schneider (Zeitschr. f. anorg. Chem. 5, 83, 1894) beobachtete Gelbfärbung elner Losung von $\mathrm{SnCl}_{4}$ in $\mathrm{SnO}_{8} \mathrm{H}_{2}$ als $\mathrm{Sn}_{2} \mathrm{O}_{3}$ anzusprechen, erscheint gewagt.

$\eta$ L. Wöhle r, Zeitschr. f. Elektr. 11, 812 (1905). g) M.van $\mathrm{Be}$ m melen, Zeitschr. $\mathrm{f}$. anorg. Chem. 23. 124 (1900). (1904). 
trockenen Substanzen waren in den meisten Fällen nicht mehr loslich, auch nicht in konzentrierter Salzsäure. Ihre Analyse - Schneider gibt darïber nichts an - wurde so ausgeführt, daf durch Glühen in Luft das Wasser ausgetrieben und im Chlorcalciumrohr direkt gewogen wurde. Der Rückstand wurde in Wasserstoff zu Metall reduziert, die Verflüchtigung von Zinn ist hierbei nicht zu befürchten, wenn die Substanz chlorfrei ist. Der Sauerstoff ergibt sich dann aus der Differenz. Der Glührückstand wird in möglichst wenig Salzsäure mit etwa $1 / 3$ Salpetersäure gelöst, und zwar zur Vermeidung einer Verflüchtigung von Zinnchlorid in einer Lintner'schen gut schließenden Druckflasche, besser im EinschluBrohr, und die Zinnsäure entweder durch längeres Erhitzen nach Zusatz von Schwefelsäure gefălt, oft und gut mit Wasser ausgekocht, geglüht und gewogen, das Platin mit Hydrazinsultat im Filtrat aus ammoniakalischer L.osung gefällt, oder besser wird es nach der Fällung der Zinnsäure mit Schwefelsäure in der Suspension elektrolytisch abgeschieden, wie in einer besonderen Abhandlung von uns ${ }^{10}$, beschrieben wird.

Der Wassergehalt ist auf die angewandte Substanz, dagegen Platin, Zinn und Sauerstoff auf Trockensubstanz berechnet worden.

Resultate:

\begin{tabular}{|c|c|c|c|c|c|}
\hline \multirow{2}{*}{$\begin{array}{r}\text { Sub- } \\
\text { stanz }\end{array}$} & \multicolumn{4}{|c|}{ Getunden } & \multirow{2}{*}{$\begin{array}{c}\text { Berechnete } \\
\text { Formel } \\
\text { Pt:Sn:O }\end{array}$} \\
\hline & $\mathrm{H}_{4} \mathrm{O}$ & $\mathrm{Pt}$ & $\mathrm{Sn}$ & 0 & \\
\hline $\begin{array}{l}1 \\
2 \\
3 \\
4 \\
5\end{array}$ & $\begin{array}{r}11,9 \\
12,4 \\
15,6 \\
10,0 \\
9,0\end{array}$ & $\begin{array}{l}14,9 \\
18,2 \\
18,3 \\
18,9 \\
20,59\end{array}$ & $\begin{array}{l}71,3 \\
64,2 \\
65,1 \\
63,5 \\
66,76\end{array}$ & $\begin{array}{l}13,8 \\
17,5 \\
17,3 \\
17,6 \\
12,6\end{array}$ & $\begin{array}{l}1: 7,84: 11,28 \\
1: 5,76: 11,67 \\
1: 5,82: 11,51 \\
1: 5,5: 11,35 \\
1: 5,80: 7,45\end{array}$ \\
\hline
\end{tabular}

Fir das Schneider'sche zinnsaure Zinnoxydui-Platinsuboxyd $\mathrm{P}_{2} \mathrm{Sn}_{4 ;} \mathrm{O}_{10}$ berechnet sich 31 Proz. Pt, 56 Proz. Sn und 13 Proz. $\mathrm{O}_{2}$, also Werte, die bei keinem der analysierten Produkte auch nur annühernd erhalten wurden, auch dort nicht, wo die Fällung, wie bei Substanz 5 , im Verhältnis von $\mathrm{Pt} z u$ Sn wie $1: 3$ stattfand. Je nach den Versuchsbedingungen entstanden andere Produkte wechselnder Zusammensetzung, die bei den Substanzen 2,3 und 4 einem Gemenge von Metazinnsäure mit metallischem Platin entspricht, während bei den Substanzen 1 und 5 , wo die Fällung momentan bezw. unter Luftabschluß stattfand, ein Gemisch mit dem Zinnoxydulhydrat vorliegt.

10) Zeitschr, I. anal. Chem. (1910).
Danach liegt es nahe, an einen Vergleich mit dem bekainten Cassi us' schen Goldpurpur zu denken, der aus Goldchlorid und Zinnchlorür entsteht und nach $H$. Debray $1 \%$. M. Mülle ${ }^{18}$ ) und E. A. Schneide ${ }^{18}$ ) eine Adsorptionsverbindung von metallischem Gold mit Zinnsäure ist, die von R. Zsigmond ${ }^{24}$ ) dann in der Tat aus kolloidem Gold und kolloider Zinnsäure mit allen Eigenschaften des Purpurs erhalten wurde. Die beiden Verbindungen gleichen sich in ihrer Löslichkeit in Salzsäure, Ammoniak und Kali, die durch Trocknen bis zur Vernichtung vermindert wird, und auch der Purpur gibt beim Eindunsten eine lösliche gelatin eähnliche Masse. Doch flockt der Goldpurpur viel schwerer als die Platinverbindung, die auch viel schneller altert und unlöslich wird, auch nicht wie jener durch Dialyse als reine wässerige Lösung 7.u erhalten ist, da sie alsbald bel Verdünnung der roten Lösung durch hydrolytisch fallende Zinnsaure ausgeflockt wird, wie ja auch kolloides Verstäubungsplatin (Bredig) weit weniger beständig ist als kolloides Gold

Die braune Platinverbindung wäre danach als Adsorptionsverbindung der beiden Kolloide des Platinmetalls und der Zinnsäure bezw. des Zinnoxydulhydrats aufzufassen, und es erklärt sich dadurch, daB mit dem schwerer hydrolisierbaren Zinnchlorür nur bei größerer Konzentration eine Fälung gleich erhalten wird, sonst erst nach Oxydation zu Zinnchlorid an der Luft, oder bei Gegenwart von Chlorid im Zinnchlorür, in neutraler Lösung sofort, in saurer erst durch Verdünnen (Hydrolyse) oder, bei Mangel an Chlorid infolge Luftabschluß, nach Zusatz von Ammoniak. Bei dem kontinuierlichen Uebergang der Rotfärbung in die schwarze Suspension mub dann auch die rote Farbe dem kolloiden Platin zugeschrieben werden, das durch Zinnsäurekolloid geschützt in Lösung erhalten wird. Infolge der freiwilligen stetigen Veränderung der Kolloide aber zur Gelbildung hin, die bekanntlich durch gewisse Salze und andere Kolloide nur beschleunigt wird, nähert es sich immer mehr dem braunschwarzen Flockungszustand. Auch beim kolloieen Gold bedentet die Farbwandlung von rot nach blau eine Annäherung an die Koagulation. Das braune kolloide Platin stellt danach die weniger fein verteilte Form dar, das rote die feiner

\footnotetext{
1) Intern Ber. 1885, 56 .

12) Journ. i. prakt. Chem. [2], 30, 360 (1884).

13) Zeitschr. f. anorg. Chem. 5, 82 (1894).

14) Liebig's Ann. 301,366 (1898).
} 
verteilte, wie die Gegenwart eines Schutzkolloids bei der Bildung des roten Platins dies nach allgemeiner Regel auch erwarten laßst und seine Deutung nach der Umhüllungstheorie ${ }^{15}$ ) in der flockungsverzögernden Reibungsverminderung durch die Zinnsäure findet, welche die primäre rote metastabile Zerteilangsstufe des Platins zu erhalten gestattet.

In Uebereinstimmung damit wird durch 1 Grammol Zinnchlorür auf 1 Grammol Platinchlorid keine Rotfärbung hervorgerufen, weil nur Platinchlorür dadurch erzeugt wird, durch 2 Grammole Zinnchlurür dagegen sofort eine schöne rote Färbung, deren Vertiefung entgegen Schneider nicht durch 3 Mol Zinnchlorïr begrenzt ist, sondern bei größerer Menge Zinnchlorür wahrscheinlich nur durch die alsdanu geringere Hydrolyse des bei der Reaktion entstehenden Zinnchlorids verlangsamt wird, wobei der Endzustand aber von der Zeitdauer sehr abhängt und durch Erhitzen wesentlich schneller erreicht wird.

Je dunkler der Niederschlag ist, um so weniger leicht ist er löslich in Salzsäure, und die mehr ins braune gehende Färbung der Lösung wird dann um so unvollkommener von Essigester oder gar Aether aufgenommen. Vielmehr werden beim Ausschütteln damit an der Grenze zwischen Aether and Wasser die braunen Flocken abgeschieden, wie allgemein nach The Svedbergin) in Acther die Kolloide instabiler sind.

Bei der Dialyse in Pergamentschlauch verhält sich die Rotfärbung in der Tat wie ein Kolloid; sie diffundiert nicht hindurch, läßt sich aber, wie erwähnt, bei weiterer Befreiung von Salzsäure nicht als klare Flüssigkeit erhalten, sondern flockt als brauner Niederschlag aus, der. sich dinn aber bei fortgesetzter Dialyse nicht wieder löst wie Zinnsilure z. B., sondern ein irreversibles Kolloid darstellt. Die negative Natur des Kolloids durch kataphoretische Wanderung der toten Substanz im U-Rohr zur Anode konnte nur sehr unvollkommen wahrgenommen werden infolge der starken anodischen Chlorentwicklung. Auch könnte dies Verhalten im Sinne der Bildung einer roten komplexen Verbindung so gedeutet werden, dak der rote Stannat-Komplex von der an sich kolloiden Zinnsäure mitgenommen wird, an ihr klebt,

15) H. Bechhold, Zeitschr. f. phys. Chem. 48, 385 (1904). Nach Billiter-Biltz wäre es die gleiche negative Ladung beider Kolloide, welche die Flockung vermindert.

16) Koll.-Zeitschr. 1, 163 (1905). wie z. B. Berlinerblau an Albumin. Auch ein Ausflocken durch Ausfrieren der roten salzsauren Lösung beim Auftauen wurde nicht wahrgenommen. Dazu kommt, dab im Verfolg der Schneider'schen Formel ${ }^{17}$ ) von uns in einer Probe der braunen Substanz in der Tat einmal unwesentliche Mengen Platinoxyd dadurch nachgewiesen wurden, dab beim Glühen derselben in Kohlendioxyd sich kleine Mengen Sauerstoff über der vorgelegten Kalilauge auffangen ließen, während doch weder Zinnoxydul noch -oxyd Sauerstoff abgeben kann. $0,7 \mathrm{~g}$ der Substanz Nr. 2 entwickelten 3,4 cem von Pyrogallol absorbierbaren Gases, bei einem zweiten Versuch entlic lien 0,46 g $2,2 \mathrm{ccm}$ Sauerstoff. Auch diffundierten kleine Mengen Platin mit der Zeit durch den Pergamentschlauch hindurch, nachweisbar durch Zinnchlorür. Ferner entwickelt die rote Lösung der Substanz in Salzsäure aus Hydroperoxyd, anders als kolloides Platin, keinen Sauerstoff, sie wird vielmehr dadurch entfärbt.

Freilich ist das Platin in der Zinusäure so fein verteilt, dafh es nach den Versuchen L. Wöhler's ${ }^{18}$ ) über die Oxydierbarkeit des Platins sich durch den Luftsauerstoff noch leichter als Platinmoor oxydieren wird, der leiclit bis zu 10 Proz. und mehr Oxyd enthalten kann. Durch die leichte Oxydation und sekundäre Chloridbildung wäre auch die geringe Menge allmäblich diffundierten Platins auterhalb des Pergamentschlauches zu erklären. Die Entfärbung der roten Lösung durch Hydroperoxyd in der salzsauren Lösung wird infolge Oxydation des Platins durch entwickeltes Chlor bewirkt, und der Mangel an katalytisch zerseizender Wirkung auf Hydroperoxyd auch in alkalischer Suspension ist durch die schützende Wirkung des Zinnoxyds hervorgerufen, wie auch das Bredig' sche Platinhydrosol durch Zusatz von kolloider Zinnsüure in seiner Wirkung auberordentlich stark beschränkt, "vergiftet “ wird, wie ich fand. Auch Formaldehyd reduziert beispielsweise das von Zinnsäure oder Gelatine geschützte Platinchlorid nicht, auch nicht nach Zusatz von Alkali. Wird Platinchlorid aber erst mit Formaldehyd und dann mit Gelatine und Alkali versetzt, so entsteht braunes kolloides Platin.

17) Auch Kane (Jour. f. prakt. Chem. 7, 35, 1836) nimmt in dem Niederschlag ein Platinoxyd an, obwohl der Sauerstoffgehalt niemals wesentlich hơher ist, als dem vorhandenen Zinn als Dioxyd entspricht, wohl aber durch den Gehalt an Zinnoxydul zuweilen niedriger. 18) Ber. d. Deutsch. chem. Ges. 36, 3484 (1903). 
Wesentlich gegen die metallische kolloide Natur der roten Lösung spricht aber vor allem die Tatsache, daB kolloides Platin bisher nur b ra u $\mathrm{n}$ b is $\mathbf{s} \mathbf{c h}$ warzerhalten worden ist durch kathodische Verstäubung sowohl wie durch Re duktion ${ }^{19}$. Aucl die Ausfallung solch kolloiden Platins durch Zinnsäurehydrosol ist braun, wie zu erwarten stand. Ebenso gewichtig spricht dagegen die Löslichkeit der roten Adsorptionsverbindung von kolloider Zinnsäure und Platin in Aether und noch mehr in Essigester, in dem beide Komponenten auch spurenweise sich nicht lösen. Hinzugefügt sei diesen zwei Punkten noch, daß auch Kaliumplatinbromid und -bromür mit Zinnbromür die gleiche Färbung gibt, die aber nach Belieben durch Erhitzen entfärbt, beim Abkühlen wieder crzeugt werden $k$ a $n$ und sich ebenso wie die Substanz aus den Chloriden in Essigester und A e ther tiefrot löst, durch größere Mengen Lösungsmittel, Aether oder Ester, aber entfärbt wird.

Der erste und gewichtigste Einwand gegen die Annahme roten Platinkolloids muBte zunåchst beseitigt werden durch die Herstellung solch roten kolloiden Platins. Dazu muete das Zinnchlorür als Reduktionsmittel ausgeschlossen und als Ersatz für das bei der Reaktion entstandene vortreffliche Schutzkolloid Zinnsäure, zugleich damit ein anderes Schutzkolloid angewandt werden. Phosphor in Aetherlösung reduziert Platinchlorid nur zu braunem Sol, im ersten Stadium aber zeigt sich eine schöne g e l b r o te Färbung, die durch ein gutes Schutzkolloid sich stabilisieren lassen mußte. Gelatine erweist sich als stärkstes Schutzkolloid, und so wurde eine einprozentige Lösung reinster Gelatine, bereitet nach Lobry de $\mathrm{Bruy}{ }^{20}$ ), mit Platinchlorür ... als $10^{-4}$ Grammollösung -.. versetzt und mit einer Losung von Phosphor in Aether durch schwaches Erwärmen unter Schütteln reduziert. Und in der Tat färbte sich die Lösung hierbei prachtvoll tiefrot. von derselben und beständigen Farbe, welche aus Platinchlorid durch Zinnchlorür entsteht, doch mit dem Stich ins Braune, den die Reduktion mit Zinnchlorür erst nach einigen Tagen erhält. In der Kälte bildet sich die Rotfärbung bereits,

19) A. Lottermoser, Ueber anorganische Kolloide, A hrens' Sammlung 6, 93 (1901). A. Gutbier, Zeitschr. f. anorg. Chem. 32, 352 (1902). C. Pa al, Ber. d. Deutsch. cliem. Ges. 37, 125 (1904). L. Garbowski ebenda 36, 1216 (1903). F. Henrich, ebenda 36, 609 (1903).

m) Chem. Centralbl. 1900, 11, 888 . wenn man das Platinchlorür als 3 bis $5 \cdot 10^{-3}$ Grammollösung, also in gröBerer Konzentration, mit Phosphor in Gelatinelösung durch Schüttein reduziert. Auch mit Wasserstoff wurde einmal gelatinehaltige Platinchloridlösung zu orangefarbigem Produkt reduziert. Meist aber erfolgt die Reduktion zu schnell und gibt dann braunes Metall, wie auch Formaldehyd dies z. B. erzeugt. Phosphor in Schwefelkohlenstoff wirkt nicht ein.

Da hierbei die rote Farbe zweifellos durch metallisches kolloides Platin hervorgerufen wird, so brauchte nur noch ihre ldentität mit jener Farbe in der Zinnchlorïrlösung festgestellt werden, am einfachsten durch das Absorptionsspektrum. Benutzt wurde dazu das sehr empfehlenswerte B e ckma n n'sche Spektroskop mit Wellenlängenskala. Die Absorption ist zweiseitig. In zwei tiefroten Lösungen gleicher Farbintensität, mit Zinnchlorür hergestellt, deren eine aber mit Gelatine versetzt war, lag das Maximum gleicher Weise zwischen 770 und $550 \mu \mu$, so daB Gelatinezusatz keine Aenderung bedingt. Durch Verdünnen wandert die $\mathrm{Ab}$ sorption, bis sie zwischen 750 und $510 \mu \mu$ auch bei weiterer Verdünnung konstant bleibt. Der Einflub von Aether als Lösungsmittel ist ohne erheblichen Einflufi, die Ränder sind aber schärfer, weshalb ein ätherischer Auszuy der Platinchloridzinnchlorürlösung mit zweifach scharfer Absorption zwischen 750 und 530 verglichen wurde mit einer in Gelatine durch ätherischen Phosphor reduzierten Platinchlorürlösung gleicher Farbintensität. - Die Absorption der letzteren zeigte einen zweiseitigen Absorptionsstreifen $z$ wischen 750 und $535 \mu \mu$, so dab die absorbierende Substanz in beiden Flässigkeiten als identisch, als rotes metallisches Platin anzusehen is t.

Wie ist nun der zweite Einwand mit diesem Befund in Einklang zu bringen, die Löslichkeit der roten Substanz aus Zinnsäure und Platinmetall in Essigester? Er erledigt sich dadurch, daß die rote Essigesterlösung als Schutzkolloid Zinnsäure gar nicht enthält. Wenn man nämlich bei Gegenwart von kolloidem Zinnsäurehydrosol, das frei ist von Chlor, Platininitrat oder eine Spur -chlorür durch ätherischen Phosphor reduziert, so erhält man, wie in Gelatinelösung, das blutrote Platin, wobei allerdings oftmals die Zinnsäure vorher ausflockt. Es ist aber in Essigester unlöslich. Als Schutzkolloid kann daher natürlich Zinnsäure in wässeriger Lösung fungieren, ist aber, wie 
durch Gelatine, auch vertretbar durch ein anderes in Essigester Iösliches Schuizkolloid, als welches man Zinnchlorid ansehen muB, das kein nennenswertes Leitvermögen hat, also undissoziiert ist ${ }^{21}$ ) und, wie bekannt ${ }^{22}$ ), undissoziiert als solches $\% \mathrm{~B}$. in Xylol äbergeht; oder aber das von $\mathrm{P}$ f e if $f$ e ${ }^{23}$ ) gefundene atherlösliche basische Chlorid, $\mathrm{SnCl}, \mathrm{OH}$, das mit $\mathrm{H}_{2} \mathrm{SnCl}_{t}$ in einer Zinnchloridlösung im Gleichgewicht sich befindet. Reduziert man nämlich Platinchlorür mit Zinnchlorür in konzentrierter Salzsăure, in welcher kolloide Zinnsäure doch nicht wohl anzunehmen ist, so tritt in gleicher Weise das rote Platin auf, wie mit Zinnsäure, das aber löslich ist in Aether. Uebrigens geht in salzsaurer Lösung auch die $R$ o t $f$ ä $\mathrm{rb}$ u $\mathrm{ng}$ durch den gelösten Cassius'schen Purpur in Aether über.

Dadurch klärt sich gleichzeitig die merkwürdige Entfärbung des roten Platins in einer Zinn bromürlösung beim Ausschïtteln mit viel Essigester dahin auf, daB das entstehende Zinnbromid im Gegensatz zu Zinnchlorid sein Brom zum Teil an Platin abzugeben vermag, dessen Bromidkomplex viel beständiger ist, als der der Zinnhromwasserstofîsäure $\left.{ }^{24}\right) \mathrm{Sn} \mathrm{Br}_{6} \mathrm{H}_{2}$, wodurch das entstehende Zinnbromür in Aether unlöslich und der Einwirkung auf das gelöste Platin entzogen wird, während $\mathrm{S}_{11} \mathrm{Br}_{3} \mathrm{OH}$ in Aether löslich ist ${ }^{25}$ ). In wässeriger Lösung sind sie derart in Gleichgewicht des Schemas

$$
\mathrm{SnBr}_{2}+\mathrm{PtBr}_{2} \underset{\text { gelb }}{\stackrel{\text { rot }}{\longrightarrow}} \mathrm{Pt}+\mathrm{SnBr}_{4} \text {, }
$$

das in der Hitze die linke Seite, beiun $\mathrm{Ab}$ kühlen die rechte beständig wird, so daß beim Erwärmen die rote Lösung gelb, beim Abkühlen die gelbe wieder rot sich färbt. In abgeschlossenen Glas entfärbt sich daher auch die rote Esterlösung nicht, wohl aber bej Zutritt von Luft.

Es ist vielleicint nicht sehr bekannt, dab auch Silbernitratlösung mit Stannonitrat in verdünnter Salpetersäure, ähnlich wie die Gold-, Platin-" und Kupferlösungen ${ }^{26}$ ) durch

9) Dittenberger und Diet $z$, Wied. Ann. 68, 857 (1899). $11907)$.

2: Smirnow, Zeitschr. 1. phys. Chem. 58, 373

\&e) Ber. d. Deutsch. chem. Ges. 38, 2466 (1905).

24 E. Bir on, Chem. Centr. 1904, II, 410.

25) Ebenda 1906, 1, 736.

26) $\mathrm{C}$. Paal und Lenze, Ber. d. Deutsch. chem. Ges. 39, 1551 (1906).
Reduktion zunächst eine blutrote Lösung gibt, die erst allnuahlich braun wird und einen rotbraunen Niederschlag fallen läBt, der nach dem Ausschütteln mit heißem Wasser von absolut identischer Farbe mit derienigen der Platinfällung wird, aber rein oxydisch ist und kein Nitrat enthält. Auch von dieser Fallung. die nach frïheren Angaben übrigens schwach explosiv sein soll, ist bisher entsprechend dem Analysenresultat angenommen worden ${ }^{27}$ ). dab eine chemische Verbindung von Silberstannat darin vorliegt, $\mathrm{Sn}_{5} \mathrm{O}_{10} \cdot \mathrm{Ag}_{2} \mathrm{O}$. 7aq., mit überschüssigem Silbernitrat aber $\mathrm{SnO}_{2} \cdot \mathrm{Ag}_{2} \mathrm{O} .2 \mathrm{aq}$, in sehr verdünnter Lösung gar ein Silbersubstannat entsteht: $\quad 5 \mathrm{SnO}_{2} \cdot \mathrm{Ag}_{4} \mathrm{O} .2 \mathrm{Sn}_{2} \mathrm{O}_{2} \mathrm{Ag}_{2} \mathrm{O} . \mathrm{n}$ aq.

Von vornherein liegt nun ein AnalogieschluB mit dem roten kolloiden Platin und seiner braunen Zinnsäureverbinulung nahe. $D a$ aber über die Analyse keinerlei Einzelheiten vorliegen, läbt sich die Art des Fehlers nicht ersehen. In meinem nitratfreien und auch nicbtexplosiven Niederschlag konnte ich jedenfalls keinen an Silber gebundenen Sauerst of entdecken, da beim Glühen in Kohlendioxyd sich absolut kein Gas entwickelte aus dem nach Vorschrift hergestellten Niederschlag. So ist auch hier nur metallisches Silber im Niederschlag anwesend, wenn auch, entsprechend der beim kolloiden Siber noch größeren Instabilităt als beim Platin, die rote Stufe nicht festzuhalten ist, wie die des Platins es war, auch nicht wie diese durch ätherischen Phosphor mit Hilfe von Gelatine oder Zinnsäure bekommen wurde, da sie von gelb sofort in braun tibergeht.

Damit erledigt sich dann auch der Analogieschlub A. D i t $f$ e's über die Bildung eines analogen "Metastannats beim Platin und Palladium", welch letzteres Metall als Chlorür mit Zinnchlorïr zunächst auch eine rotgelbe, bald aber eine grüne Lösung erzeugt.

Die schwarze Fällung von Silberlösung durch Zinnchlorür in Alkali ist bereits früher von Pillitz ${ }^{28}$ ) als Gemisch von Metall und 7inusäure erkannt worden - eine Lösung des schwarzen Silber,purpurs" ist von A. Lot termoser ${ }^{29}$ ) dargestelt - wie es auch be $i$ der Einwirkung von Zinnchlorür auf Wi nut=, Quecksilbeı- und Kupferlösung entsteht

27) A. Ditte, Jahres-Bericht 1882, 1301; Ann. Phys. Chim. [5] 27, 171 (1882).

28) 7 itschr. f. anal. Chem. 21,27 (1882).

29) A hrens, Sammlung chem. und chem.-techn. Vorträge 6, 213 (1901). 
Zus ammenf assung:

1. Die Rotfärbung, welche Zinnchlorür mit Platinlösung hervorruft, ist nicht durch die Bildung von Platinchlorïr bedingt; sie wurde erkanut als kolloides Platinmetall, das durch Schutzkolloide wie. Zinnchlorid und seine kolloiden Hydrolysenprodukle in dieser ungewöhnlich feinen Verteilung sich für eine Zeit festhalten läit, ohne dieselben aber sofort in dis braune Kolloid ïbergeht.

2. Das rote kolloide Platin wurde auch durch ein anderes Reduktionsmittel, nämlich Phosphortösung bei Gegenwart eines anderen Schutzkolloids, nämlich Gelatine, erhalten, ihre Identität durch Absorptionspektrum festgestellt.

3. Die schokoladenbraune Fallung, welche durch Hydrolyse des entstehenden Zinnchlorids in der roten Lösung sich bildet, erwies sich entgegen bisheriger Annahme nicht als eine
Verbindung von Zinnsesquioxyd und Platinsuboxyd, sondern als das Analogon des Cassius schen Goldpurpurs, als ein Gemenge von Zinnsäure und Platinmetall in wechselndem Verbältnis je nach den Versuchsbedingungen. Der Platin "purpur" hat die analogen chemischen Eigenschaften des Goldpurpurs.

4. Als neue Eigenschaft wurde die Löslichkeit in Essigester und Aether erkannt und das Verhalten dieser Lösungen untersucht.

5. Die D it te sche Reaktion der Rotfärbung von Silbernitrat durch Stannonitrat wurde entgegen seiner Annahme nicht als eine Verbindung des Silberoxyds bezw. -suboxyds der Zinnsäure, sondern als analoge Bildung eines Silber. .purpurs" erkannt.

Herrn Dr. Ing. F. Martin danke ich bestens für die zuverlässige Ausführung einiger quantitativer Bestimmungen.

\section{Ueber kolloides Zirkoniumsilizid.}

Von E. Wedekind.

(Eingegangen am 1. Oktober 1910)

\section{(Dritte Mitteilung ${ }^{1)}$ (iber die Darstellung von Hydrosolen durch Anatzung.)}

Die Herstelling von kolloiden Stoffen durch chemische Zerstäubung mit Säuren, die ich beim Zirkonium ${ }^{2}$ ) und beim Thotium ${ }^{3}$ ) beschrieben habe, stellt gewissermaßen ein Spezialverfahren der allgemeinen Arbeitsweise dar, welche H. Kuz eli4) in seinen Patenten zur Gewinnung von kolloiden Metallen niedergelegt hat; dieselbe besteht bekanntlich darin, dat die bereits auf mechanischem Wege sehr fein verteilten Materialien abwechselnd so lange mit sauren und alkalischen Reagenzien angeätzt werden, bis sie in den kolloiden Zustand übergegangen sind.

Ich habe nun diese Anätzmethode, welche sich meistens gut bewährte bei Elcmentarstoffen, die gegen Säuren bezw. Alkalien relativ widerstandstähig sind, auf einfache Verbindungen der seltenen Metalle mit Silizium bezw. Bor angewandt. Diese Silizide und Boride haben durchweg metallischen Charakter, zeichnen sich durch ziemliche Widerstandsfähig-

4) Diese Mitteilung erscheint gleichzeitig in der Festschrift zum van Bem melen-Jubilàm.

7) Koll.-Zeitschr. 2, 289 (1908).

6) E. Wedekind und H. Baumhauer, ibid, 5 , $191(1909)$ : inzwischen ist' auch die Kolloidisierung des Titanmetalles gelungen.

4) Vgl. hierzu den Aufsatz von A. Lottermoser in der Chem.-Ztg. 1908, Nr. 25 keit aus und sind zum Teil relatıv leicht zugänglich. Als besonders geeignetes Objekt erwies sich zunächst das Zirkoniumsilizid $\mathrm{ZrSi}_{2}$. Dieses Silizid ist schon von O. Hönigschmidt ${ }^{5}$ beschrieben worden: ich selbst habe vor längerer Zeit über Versuche berichtet, eiı Zirkonsilizid $\mathrm{ZrSi}$ durch Einwirkung von freiem Silizium auf Zirkonoxyd bei den Temperaturen des elektrischen Lichtbogenofens darzustellen ${ }^{\circ}$ ). Die nähere Untersuchung hat inzwischen ergebeli, dab das auf elektrothermischen Wege erhaltene Produkt in wesentlichen nach der Formel $\mathrm{ZrOSi}$ zusammengesetzt ist, also ein Zirkonoxydsilizid darstellt.

Am besten gewinnt man das Zirkonsilizid $\mathrm{ZrSi}_{2}$ durch Einwirkung von ïberschüssigem Silizium auf Zirkonkaliumfluorid $\mathrm{K}_{2} \mathrm{ZrF}_{8}$ im elektrischen Ofen. Da der Ofen, welcher mir seinerzeit zur Verfügung stand, nur kleine Materialmengen $z u$ verarbeiten gestattete, so wandte ich mich an die Deutsche Goldund Silberscheideanstalt in Frankfurt a. M., welche mir in liberaler Weise ihre groBen elektrischen Oefen zur Verfügung siellte. Hier konnte ich gröbere Mengen des Silizides dar-

5) Monatsh. f. Chem. 27, 1074.

6) Ber. d. Deutsch. chem. Gies. 35, 3932 (1902). 